\title{
SOLAR-LIKE OSCILLATIONS AND ACTIVITY IN PROCYON: A COMPARISON OF THE 2007 MOST* AND GROUND-BASED RADIAL VELOCITY CAMPAIGNS
}

\author{
Daniel Huber $^{1}$, Timothy R. Bedding ${ }^{1}$, Torben Arentoft $^{2}$, Michael Gruberbauer ${ }^{3}$, David B. Guenther ${ }^{3}$, \\ GÜnter HoudeK $^{4}$, Thomas Kallinger ${ }^{4,5}$, Hans KJeldsen $^{2}$, Jaymie M. Matthews ${ }^{5}$, Dennis Stello ${ }^{1}$, and Werner W. Weiss ${ }^{4}$ \\ ${ }^{1}$ Sydney Institute for Astronomy (SIfA), School of Physics, University of Sydney, NSW 2006, Australia; dhuber@physics.usyd.edu.au \\ ${ }^{2}$ Danish AsteroSeismology Centre (DASC), Department of Physics and Astronomy, Aarhus University, DK-8000 Aarhus C, Denmark \\ ${ }^{3}$ Institute for Computational Astrophysics, Department of Astronomy and Physics, Saint Marys University, NS B3H 3C3, Halifax, Canada \\ ${ }^{4}$ University of Vienna, Institute for Astronomy, 1180 Vienna, Austria \\ ${ }^{5}$ Department of Physics and Astronomy, University of British Columbia, 6224 Agricultural Road Vancouver, BC V6T 1Z1, Canada \\ Received 2010 October 19; accepted 2011 February 11; published 2011 March 29
}

\begin{abstract}
We compare the simultaneous 2007 space-based MOST photometry and ground-based radial velocity (RV) observations of the F5 star Procyon. We identify slow variations in the MOST data that are similar to those reported in the RV time series and confirm by comparison with the Sun that these variations are likely the signature of stellar activity. The MOST power spectrum yields clear evidence for individual oscillation frequencies that match those found in the RV data by Bedding et al. We identify the same ridges due to modes of different spherical degree in both data sets, but are not able to confirm a definite ridge identification using the MOST data. We measure the luminosity amplitude per radial mode $A_{l=0 \text {,phot }}=9.1 \pm 0.5 \mathrm{ppm}$. Combined with the estimate for the RV data by

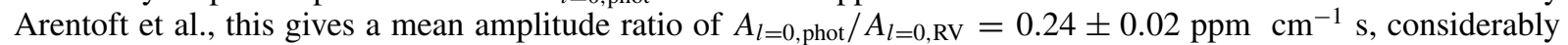
higher than expected from scaling relations but in reasonable agreement with theoretical models by Houdek. We also compare the amplitude ratio as a function of frequency and find that the maximum of the oscillation envelope is shifted to higher frequencies in photometry than in velocity.
\end{abstract}

Key words: stars: activity - stars: individual (Procyon) - stars: oscillations - stars: rotation - techniques: photometric - techniques: radial velocities

Online-only material: color figures

\section{INTRODUCTION}

The detection and measurement of oscillations in stars provides a unique possibility to infer details about the physics governing their interiors. The prospect of extending such studies from the Sun to distant stars has motivated many observation campaigns in recent decades. Even with the wealth of new space-based photometry from CoRoT (see, e.g., Michel et al. 2008) and Kepler (see, e.g., Gilliland et al. 2010), there is still an important place for ground-based spectroscopic campaigns of bright nearby stars with well-known fundamental parameters. Owing to its brightness $(V=0.3)$, proximity $(d=3.5 \mathrm{pc})$, and membership in an astrometrically well-determined binary system, the F5 sub-giant Procyon A ( $\alpha$ CMi, HR 2943, HD 61421) has long been considered a prime target for such campaigns.

The majority of early efforts to detect oscillations in Procyon have relied on measuring Doppler velocities from a single site. The first claimed detection dates back to Gelly et al. (1986) which, however, could not be confirmed by Libbrecht (1988) or Innis et al. (1991), who reported null-detections at similar sensitivity levels. With the benefit of hindsight, it now seems that the first detection of power excess in Procyon (and, in fact, any other solar-like star than the Sun) was by Brown et al. (1991). This was followed by numerous observing campaigns, mostly single-site, taking advantage of the increasing precision of Doppler-shift measurements (Mosser et al. 1998; Barban et al. 1999; Martić et al. 1999, 2004; Claudi et al. 2004; Eggenberger et al. 2004; Bouchy et al. 2004; Leccia et al. 2007). While all of these studies revealed clear power excess

\footnotetext{
* Based on data from the MOST satellite, a Canadian Space Agency mission, jointly operated by Dynacon Inc., the University of Toronto Institute for Aerospace Studies and the University of British Columbia, with the assistance of the University of Vienna.
}

in the expected frequency range of $0.5-1 \mathrm{mHz}$, a consistent determination of individual frequencies was hampered due to severe aliasing caused by daily gaps inevitable in dual- or singlesite observations.

The first two sets of continuous observations of Procyon by the Canadian Microvariability and Oscillations of STars (MOST) satellite (Walker et al. 2003; Matthews 2007) in 2004 and 2005 resulted in null-detections, leading to the conclusion that luminosity amplitudes in Procyon must be lower than $15 \mathrm{ppm}$ and/or the mode lifetimes shorter than 2-3 days (Matthews et al. 2004; Guenther et al. 2007). Bedding et al. (2005) found these results to be compatible with limits set from ground-based radial velocity (RV) observations and Baudin et al. (2008) confirmed the null-result, while Régulo \& Roca Cortés (2005) and Marchenko (2008) cautiously claimed a detection of oscillations based on a re-analysis of MOST data. Meanwhile, Bruntt et al. (2005) reported a detection of power excess with amplitudes of about twice the solar value $(\sim 8 \mathrm{ppm})$ based on continuous space-based photometry by the WIRE satellite, consistent with the upper limits set by the MOST results.

The clear identification of individual oscillation modes in Procyon was finally achieved with a large ground-based RV campaign that was carried out in 2007 January (Arentoft et al. 2008; Bedding et al. 2010). Simultaneously, a third set of MOST observations, longer and with higher precision than the previous runs, was obtained in 2007 January and February (Guenther et al. 2008). Here, we present the first direct comparison of these data sets.

\section{SUMMARY OF OBSERVING CAMPAIGNS}

The following sections present a brief summary of the observations and main results of the two campaigns on which 
the comparison in this paper is based. For a brief introduction to basic characteristics of solar-like oscillations, relevant to Procyon, we refer the reader to Section 2 in Bedding et al. (2010).

\subsection{MOST Photometry}

The MOST space telescope, launched in 2003, is the first satellite dedicated to asteroseismic observations from space. MOST houses a $15 \mathrm{~cm}$ telescope with observations performed through a custom broadband filter $(350-700 \mathrm{~nm})$. It is positioned in a sun-synchronous low-Earth orbit, enabling it to continuously monitor stars for up to three months. For a detailed description of the instrument, we refer to Walker et al. (2003).

Guenther et al. (2008) presented the results of the MOST 2007 campaign. The data are the most precise MOST Procyon photometry to date, outperforming the previous runs in both length (38.5 days) and time-series point-to-point scatter (140 ppm), and consequently also in high-frequency noise $(0.9 \mathrm{ppm})$. While the power spectrum showed an excess in the expected oscillation frequency range and an autocorrelation of the spectrum yielded strong evidence for the expected characteristic large frequency separation $(\Delta v)$ of $\sim 55 \mu \mathrm{Hz}$, no reliable individual mode frequencies could be extracted. Using various common and new frequency extraction tools, Guenther et al. (2008) found that no consistent regularly spaced frequencies could be identified unless it was assumed that the mode lifetimes are $\sim 2$ days, i.e., slightly less than solar (see, e.g., Chaplin et al. 2009). The large scatter of the extracted frequencies around the predicted regularity $( \pm 5 \mu \mathrm{Hz}$ ) was found to be consistent with the scatter of the frequency identifications from previous RV campaigns. Using new numerical convection models, Guenther et al. (2008) argued that, contrary to the Sun, the granulation timescale in Procyon is similar to the timescale of the $p$-mode oscillations. This was identified as a possible reason for the short mode lifetimes in Procyon and the consequent difficulty of extracting consistent frequencies from the MOST 2007 data.

\subsection{Ground-based Radial Velocity Campaign}

The 2007 ground-based RV campaign was described in detail by Arentoft et al. (2008) and included 11 telescopes, with apertures ranging from 0.6 to $3.9 \mathrm{~m}$, at eight observatories. Covering a total length of 25 days, with a duty cycle above $90 \%$ for the central 10 days of observations, it is the most precise and complete RV campaign dedicated to asteroseismology to date.

Arentoft et al. (2008) reported the detection of slow variations in the velocity time series with an apparent period of $\sim 10$ days, which they attributed to rotational modulation of active regions on the surface of the star. A possible rotation period of Procyon of $\sim 10$ days or twice that value was suggested, with the latter scenario being more likely if it is assumed that the rotation axis is aligned with the known inclination of the binary orbit. Arentoft et al. (2008) also provided an estimate of the mean mode amplitude between 650 and $1150 \mu \mathrm{Hz}$ of $38.1 \pm 1.3 \mathrm{~cm} \mathrm{~s}^{-1}$, consistent with previously reported detections and upper limits.

A detailed asteroseismic analysis of the RV data was presented by Bedding et al. (2010). The continuous coverage and high signal-to-noise ratio $(\mathrm{S} / \mathrm{N})$ allowed the first measurements of mode frequencies in Procyon. These authors also measured large and small frequency separations as a function of frequency and identified a possible mixed mode at $446 \mu \mathrm{Hz}$. They used the variation of peak amplitudes caused by the stochastic nature of the oscillations to estimate the mode lifetimes in Procyon to be $1.3 \pm 0.5$ days. As a result of the large linewidths of the modes, however, a major difficulty in the analysis was the identification of modes with even and odd spherical degree $l$. Bedding et al. (2010) gave several arguments for the most probable mode identification but left open the possibility that the identification could be reversed.

\section{SLOW VARIATIONS}

The Procyon MOST photometry provides the opportunity to further investigate the nature of the slow variations detected in RV. The stability and continuity of MOST photometry has previously been successfully used to study activity in several stars (Croll et al. 2006; Walker et al. 2007).

The analysis by Guenther et al. (2008) was based on a highpass filtered MOST light curve to focus on the detection of solar-like oscillations. Here, we use the raw light curve, as produced by the reduction pipeline described in Reegen et al. (2006), as the starting point of our analysis. As discussed by Huber et al. (2009b) for another solar-like star observed by MOST (85 Peg), the reduced photometry sometimes shows long-periodic instrumental variability that can be identified and corrected by decorrelating satellite telemetry data, such as board and preamplifier temperatures, against the observed target intensities. We applied the same technique to the 2004, 2005, and 2007 photometry of Procyon and the resulting detrended light curves are shown in Figure 1.

The intensity and velocity curves in Figure 1 show similar variability in all data sets. The most prominent periodicity in the velocities, with a period of $\sim 10$ days, is not readily apparent in the 2007 MOST photometry, which shows the strongest signal as measured from the amplitude spectrum with a period of $\sim 6$ days. However, as is well known for the Sun and demonstrated by Clarke (2003) for models of spotted stars, the relationship between simultaneous velocity and intensity observations of active regions is not simple and strongly depends on parameters such as rotational velocity, inclination, and spot size. Detailed modeling of the variations using the overlapping parts of the data set ( $\sim 10$ days) is beyond the scope of this paper, but we make some qualitative statements on the variability based on the observed low-frequency power levels.

To compare both data sets independent of length and sampling, we converted the power spectra to power density by multiplying with the effective length of each data set (calculated as the inverse of the area under the spectral window in power). Figure 2 shows the low-frequency power density level measured in Procyon in intensity (upper panel) and velocity (lower panel) using the full length of both data sets obtained in 2007. To measure the low-frequency power density level, we fitted power laws with a fixed slope of two in the frequency interval 5-40 $\mu \mathrm{Hz}$ (red dashed lines). For comparison, the blue dashed-dotted lines show the average power density level of the Sun during solar maximum derived using the same method with 30 day subsets of data obtained in intensity by VIRGO (Fröhlich et al. 1997) and in velocity by GOLF (Ulrich et al. 2000; García et al. 2005), both of which are instruments onboard the Solar and Heliospheric Observatory $(\mathrm{SOHO})$ spacecraft. Note that we have corrected the photometric power density for the spectral response of the MOST filter following the method of Michel et al. (2009), yielding $R_{\mathrm{g}}=4.23$ using an effective temperature of $T_{\text {eff }}=6500 \mathrm{~K}$ for Procyon, compared to $R_{\mathrm{g}}=5.02$ for solar VIRGO observations in the green channel.

As noted by Arentoft et al. (2008), the Procyon velocity power spectrum shows a similar $1 / \nu^{2}$ dependence as observed for the 

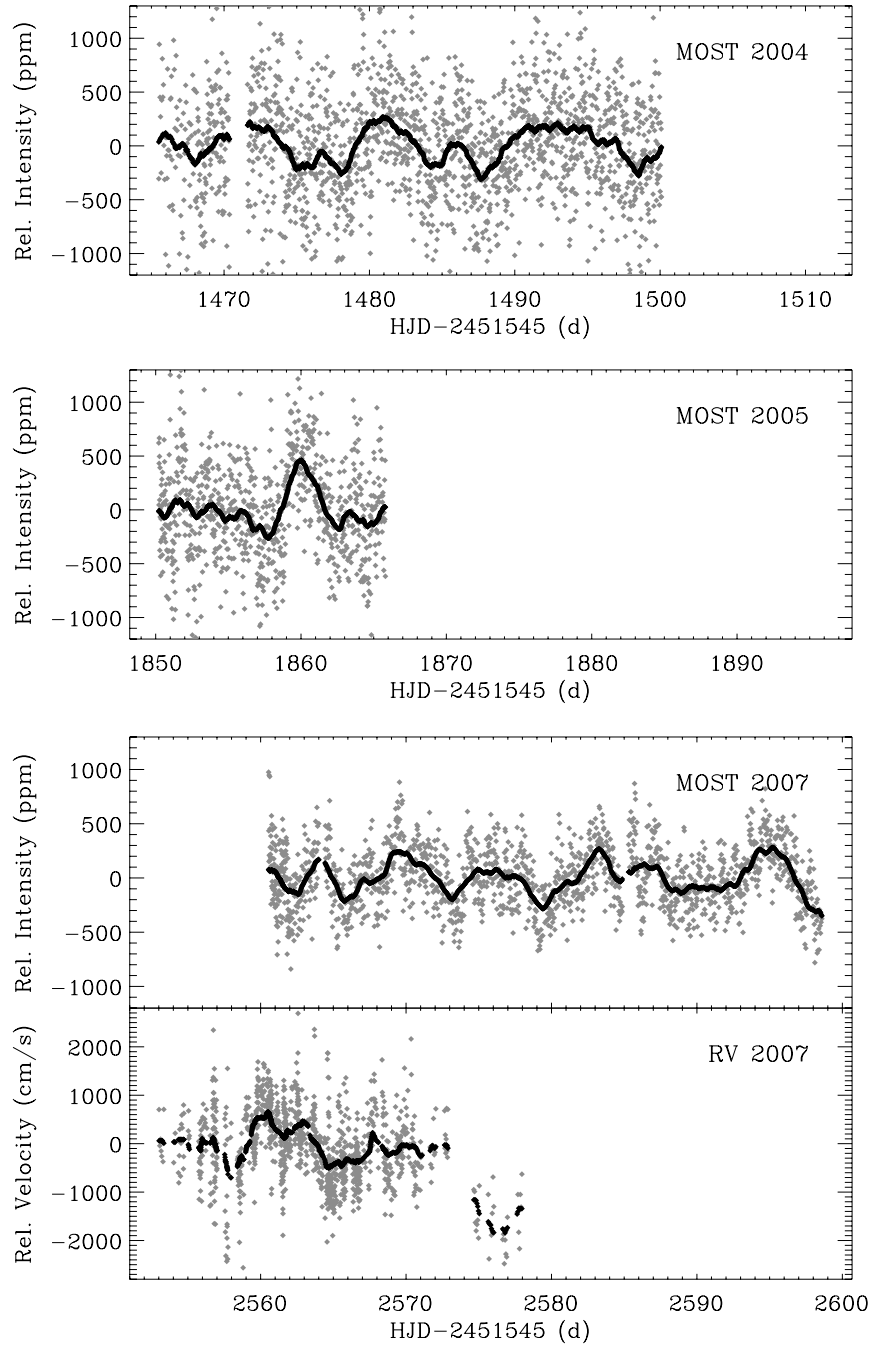

Figure 1. Detrended MOST light curves of Procyon obtained in 2004, 2005, and 2007 (every 50th data point shown) and 2007 radial velocity curve (every 20th data point shown) of Procyon. The thick black lines show the result of smoothing with a boxcar of width $\sim 2$ days.

Sun but at higher power density levels and the same is observed in the photometry. Is the observed excess of photometric power density, measured relative to the Sun, consistent with the velocity observations? To investigate this, we used $\mathrm{SOHO}$ data spanning from 1996 to 2004 and measured the low-frequency power density levels in independent 30 day subsets as described above throughout the solar activity cycle. The ratios of the observed levels between Procyon and the Sun for each subset are shown in Figure 3(a).

We observe that the power density ratio between Procyon and the Sun is substantially higher in velocity than in photometry. Arentoft et al. (2008) argued that the velocity power density at frequency $v$ is expected to scale as follows:

$$
\mathrm{PD}(v)_{\mathrm{RV}}=\left(\frac{d a}{a}\right)^{2}\left(\frac{v \sin i}{T}\right)^{2} v^{-2},
$$

where $d a / a$ is the fractional area covered by active regions, $v \sin i$ is the projected rotational velocity, and $T$ is the typical lifetime of active regions on the stellar surface.

Similarly, the amplitude measured in photometry will be proportional to $d a / a$ and the luminosity variation $\delta L / L$ caused by the flux contrast between the unspotted and spotted areas

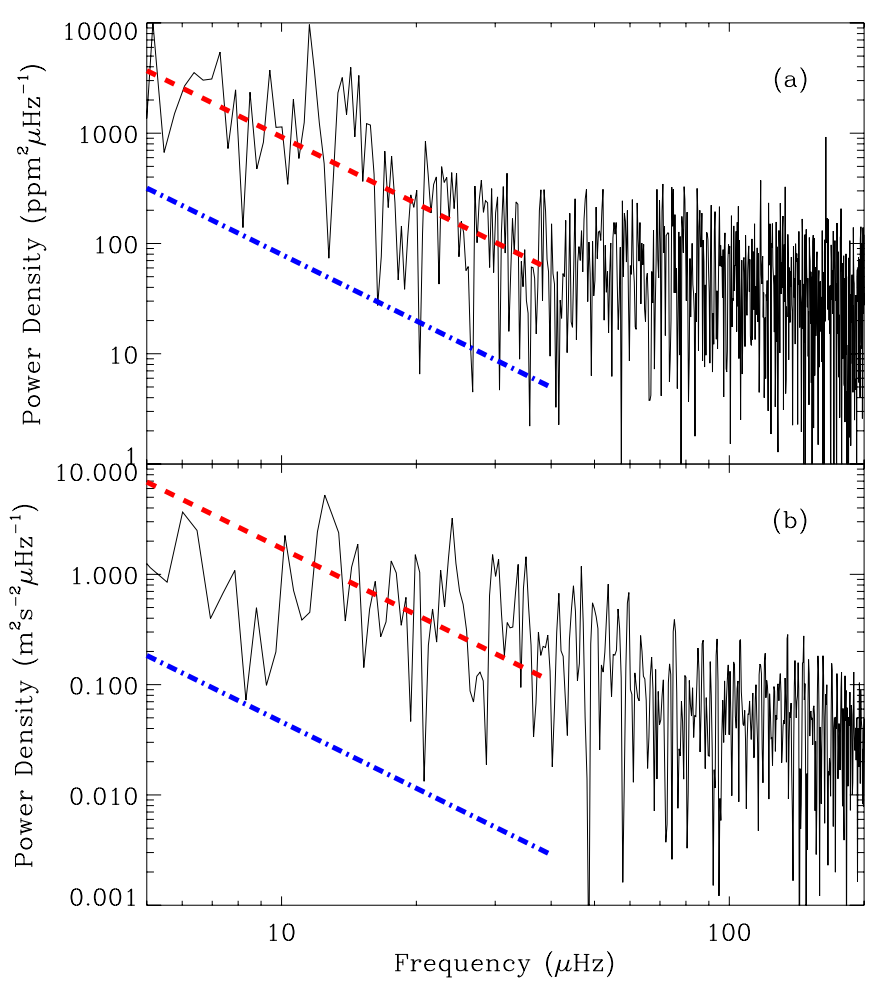

Figure 2. (a) Photometric power density spectrum of Procyon. The red dashed line shows a power law with a fixed slope of two fitted in the frequency interval 5-40 $\mu \mathrm{Hz}$. The blue dashed-dotted line shows the average power density level of the Sun during solar maximum, determined using the same method with 30 day subsets of $\mathrm{SOHO}$ data. (b) Same as panel (a) but for radial velocities.

(A color version of this figure is available in the online journal.)

of the star (see, e.g., Dorren 1987). We can therefore rewrite Equation (1) for the case photometric power densities as

$$
\mathrm{PD}(v)_{\text {Phot }}=\left(\frac{d a}{a}\right)^{2}\left(\frac{\delta L / L}{T}\right)^{2} v^{-2} .
$$

We note that Equation (2) is only intended to give an approximate estimation of the photometric power density due to stellar activity. Compared to detailed photometric spot models (see, e.g., Dorren 1987; Lanza et al. 2003; Mosser et al. 2009), Equation (2) for example does not include an explicit dependence on stellar inclination. As shown by Mosser et al. (2009), this corresponds to neglecting any information about the latitude $\lambda$ of active regions (i.e., we assume in Equation (2) that it is equally likely to observe spots near the equator or near the poles). While this is certainly not the case for the Sun, this information is also neglected in Equation (1) (e.g., an active region at $\lambda=90^{\circ}$ on a star with $i=90^{\circ}$ will cause no velocity variation). Since we are here only interested in comparing the ratio of velocity to photometry variations, any explicit dependence on the latitude of active regions will therefore cancel out.

Equations (1) and (2) imply that the difference between the velocity and photometry power density ratio of two stars depends only on $v \sin i$ and $\delta L / L$. We assume that the ratio in $\delta L / L$ (and hence the flux contrast) between Procyon and the Sun is negligible compared to the ratio in $v \sin i$, which is supported by detailed spot modeling of stars hotter than the Sun (Lanza et al. 2009, 2011). Using $v \sin i=2.0 \mathrm{~km} \mathrm{~s}^{-1}$ for the Sun and $v \sin i=3.2 \mathrm{~km} \mathrm{~s}^{-1}$ for Procyon (Allende Prieto et al. 2002), this therefore implies a difference in the velocity and photometry power density ratios by a factor of $\sim 2.56$. The red dashed-dotted 

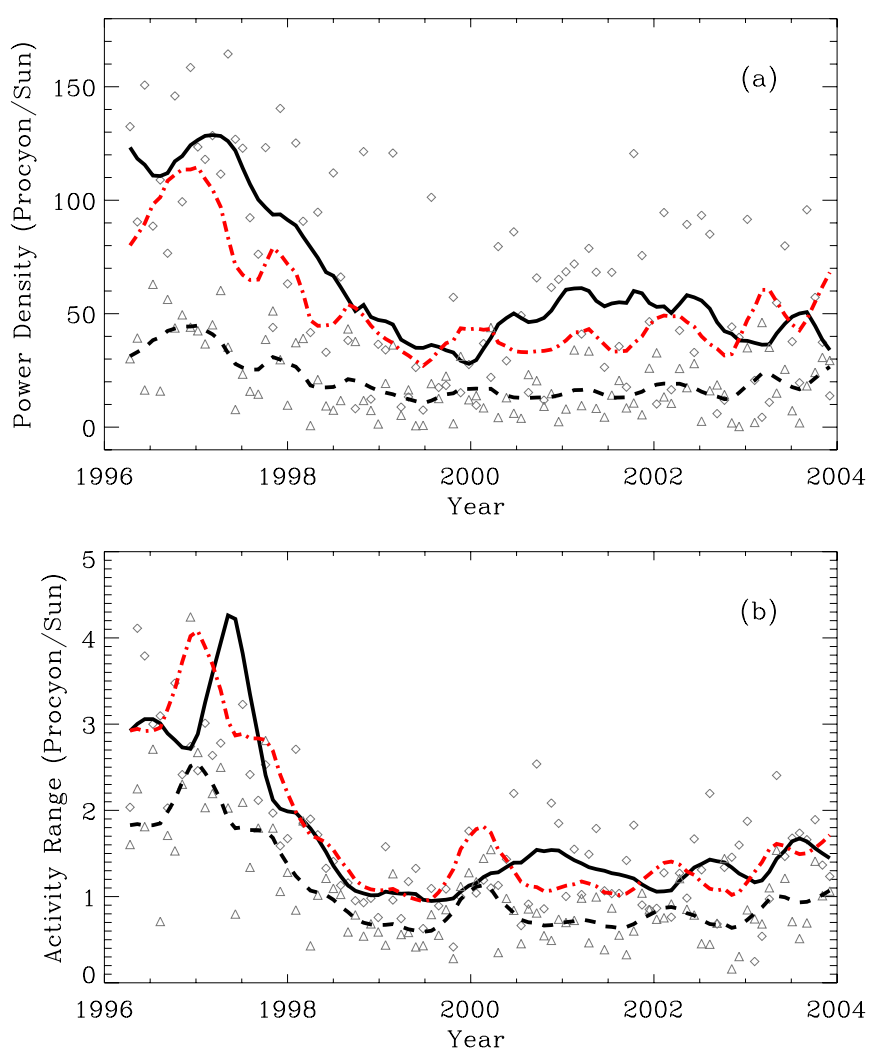

Figure 3. (a) Ratio of low-frequency power density for Procyon and the Sun as a function of solar activity cycle in photometry (triangles, dashed line) and velocity (diamonds, solid line). Open symbols show individual measurements using 30 day subsets and lines are values which were smoothed twice using a boxcar with a width of $\sim 5$ months. The red dashed-dotted line shows the photometric ratio scaled to account for the different projected rotational velocities of Procyon and the Sun. (b) Same as panel (a) but using the activity range as defined in Basri et al. (2010).

(A color version of this figure is available in the online journal.)

line in Figure 3(a) shows the photometric power density ratio multiplied by this value. We observe that the power density ratios are now in better agreement, both during solar minimum (in 1996) and solar maximum (in 2002). This result implies that the observed variations of Procyon in both data sets are qualitatively in agreement with being the signature of stellar activity.

The scatter of individual data points in Figure 3(a) is large, presumably due to the complex non-sinusoidal variations of stellar activity causing large variations in low-frequency power density fits. We have therefore repeated the above exercise using the activity range as defined in Basri et al. (2010), which measures the maximum absolute deviation of the time series with respect to its mean. The results of this are shown in Figure 3(b). In this case, the scaling factor is expected to be $\sqrt{2.56}$. Again, the scaled photometric ratios are in reasonable agreement with the velocity ratios, confirming the results derived using power densities.

\section{SOLAR-LIKE OSCILLATIONS}

\subsection{Comparison of Power Spectra}

Figure 4 compares the power spectra of both full 2007 data sets in the frequency range where $p$-modes have been detected. We used the sidelobe-optimized power spectrum for the velocities, as described by Bedding et al. (2010) and the highpass filtered MOST data used by Guenther et al. (2008). Note that the funnels of low power around the orbital harmonics of MOST (marked as dashed lines) are due to the high-pass filter removing power leaking from low frequencies, which in turn is caused by periodic outlier rejections in the data reduction pipeline during high-straylight phases. To smooth over the effects caused by the stochastic nature of the oscillations, we convolved both spectra with a Lorentzian profile with a width of $2.5 \mu \mathrm{Hz}$, corresponding to a mode lifetime of 1.5 days. We also show, with dotted lines, the values for the odd and even ridge centroids determined by Bedding et al. (2010) for the velocity data (see their Figure 9). Note that although our adopted value for the mode lifetime is larger than measured for other F-stars such as HD 49933 (Gruberbauer et al. 2009; Benomar et al. 2009), the exact choice of this value has no influence on the results presented below.

The comparison shows clearly that most of the peaks in the $M O S T$ spectrum coincide with pulsation frequencies identified

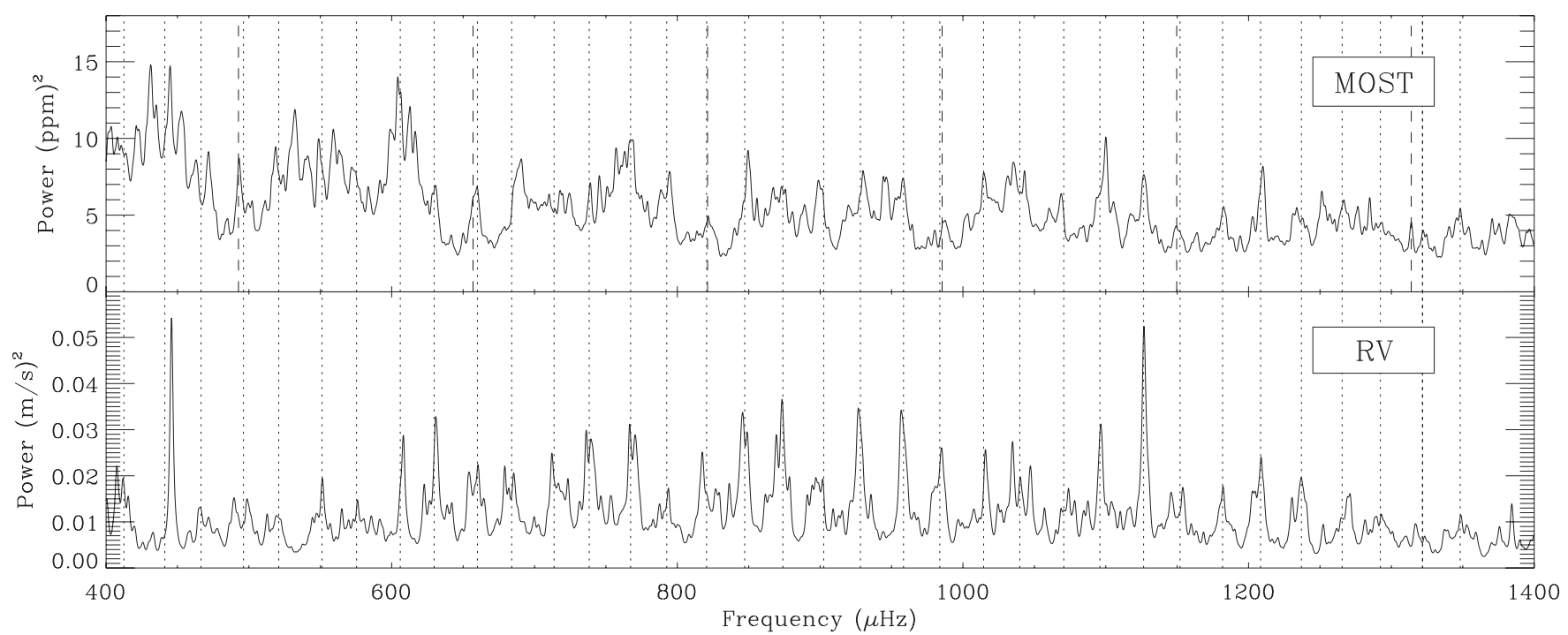

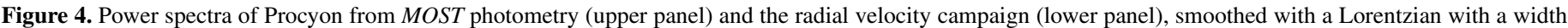

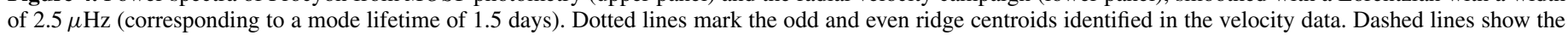
harmonics of the MOST orbital frequency. 


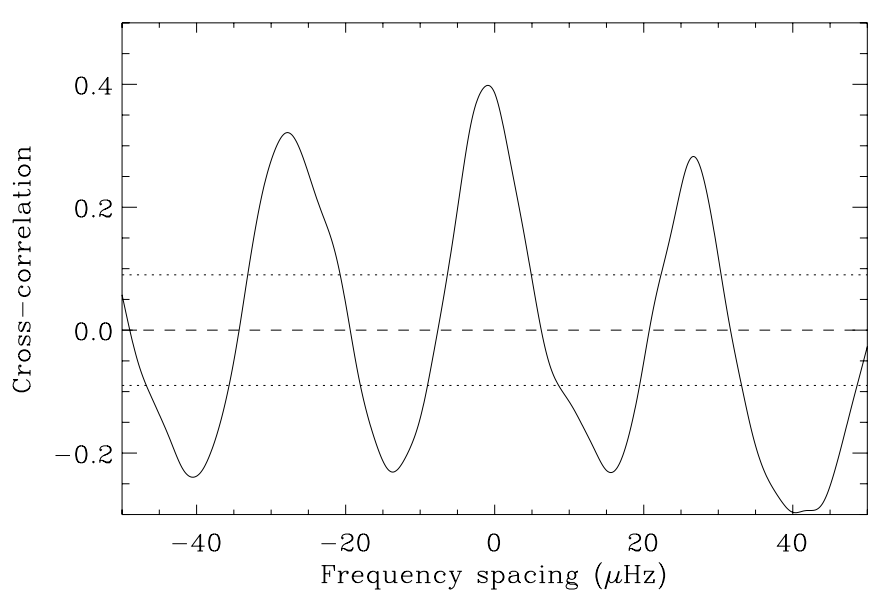

Figure 5. Cross-correlation of the velocity and photometry power spectra in the frequency range $650-1150 \mu \mathrm{Hz}$. The dashed and dotted lines mark the mean and $\pm 1 \sigma$ noise level derived from cross-correlating the RV power spectrum with 2000 MOST power spectra computed from white noise time series with the same sampling and scatter as the original data.

in the velocity power spectrum. The agreement improves considerably toward high frequencies $(>800 \mu \mathrm{Hz})$, where the granulation background in photometry becomes lower, as can be seen by the steady decrease of power toward high frequencies (see also Section 4.3). We also see that, unluckily, several of the intrinsic pulsation frequencies of Procyon near maximum power coincide almost exactly with harmonics of the orbital frequency of the MOST satellite.

To investigate the agreement between the two spectra in more detail, we calculated a cross-correlation of the two power spectra in the central region of maximum power from $650-1150 \mu \mathrm{Hz}$. The result is shown in Figure 5. As expected, we see clear maxima at zero offset and at half the large frequency separation. Note that the shift of the highest peak from zero offset is only $0.9 \mu \mathrm{Hz}$, which is much smaller than the lifetime of the modes and therefore insignificant. To test the significance of the peak height, we correlated the RV power spectrum with MOST power spectra calculated from 2000 white noise time series with the same sampling and scatter as the original data set. The resulting distribution at zero offset showed a mean of zero and a standard deviation of 0.09, which is indicated as dashed and dotted lines in Figure 5. At a level of $>4 \sigma$, these results confirm that the peaks observed in the MOST power spectrum very likely correspond to the oscillations observed in the RV data.

\section{2. Échelle Diagrams and Folded Power Spectra}

A widely used method to analyze the regular frequency pattern characterizing solar-like oscillations is to stack the power spectrum (or extracted frequencies) in slices of the large frequency separation $\Delta v$, forming a so-called échelle diagram (Grec et al. 1983). Note that throughout the paper we use $\Delta v=56 \mu \mathrm{Hz}$, which corresponds to the large separation at maximum power as identified in the RV data (see Figure 11(a) in Bedding et al. 2010). The left and middle panels of Figure 6 show échelle diagrams of both power spectra smoothed to the same frequency resolution. Note that the MOST power spectrum has been corrected for the background contribution due to granulation and activity (see Section 4.3). The échelle diagrams clearly show two ridges corresponding to modes of odd and even degree in both data sets. The similarity of the curvature of both ridges (and hence the variation of $\Delta v$ with frequency) in both individual data sets reaffirms our conclusion that the peaks seen in Figure 4 are intrinsic $p$-modes. The échelle diagrams also show that the higher low-frequency noise in the photometry makes it harder to detect $p$-modes below $\sim 800 \mu \mathrm{Hz}$ than in the velocity data.

In addition to the MOST and RV data sets, we also analyzed a power spectrum constructed by multiplying both individual power spectra. This corresponds to a power spectrum of the convolution of the MOST and RV time series, combining the advantage of the higher frequency resolution of the MOST data with the higher $\mathrm{S} / \mathrm{N}$ of the RV data. The resulting échelle diagram displayed in the right panel of Figure 6 clearly shows more well-defined ridges than the corresponding diagrams using the individual data sets.

Having confirmed that we have detected the ridges in both data sets, an obvious step in the analysis is to attempt to confirm or discard the ridge identification presented in Bedding et al. (2010) using the MOST data. It is well known that

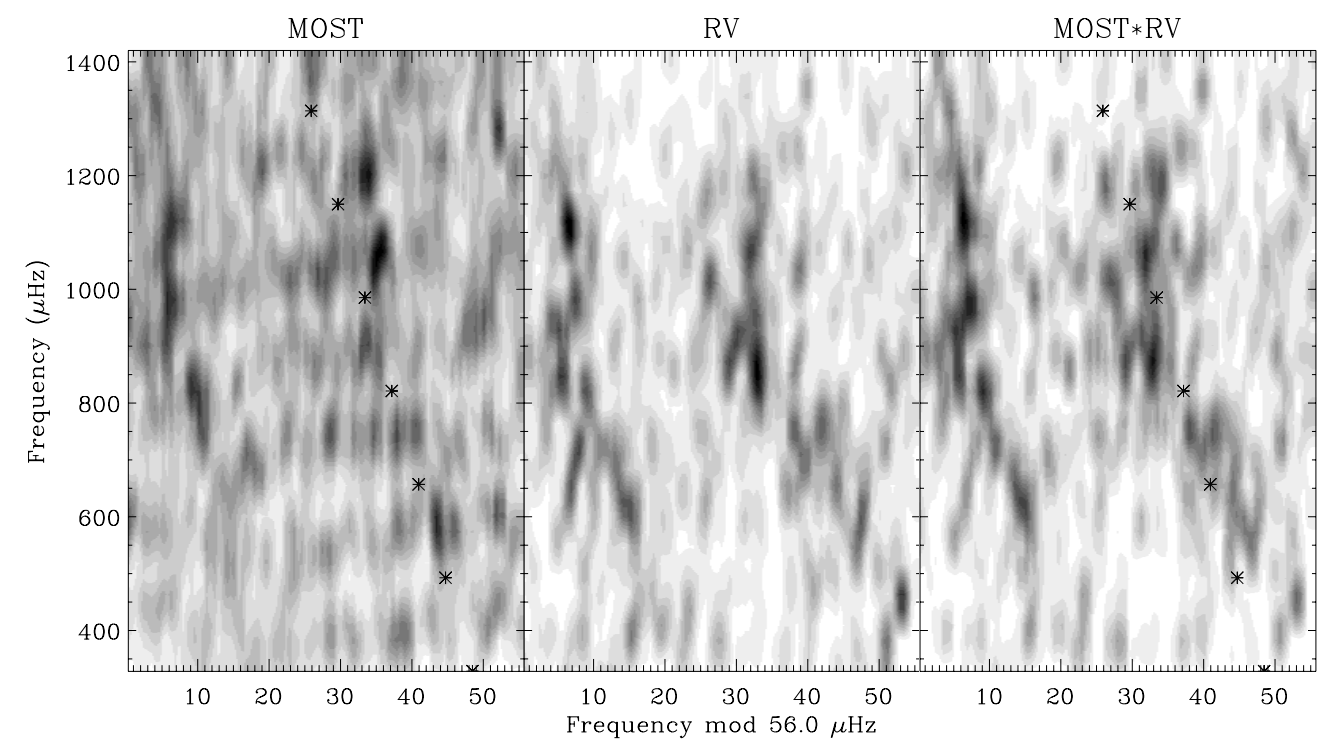

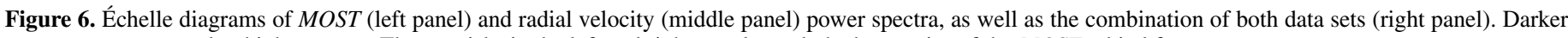
gray tones correspond to higher power. The asterisks in the left and right panels mark the harmonics of the MOST orbital frequency. 


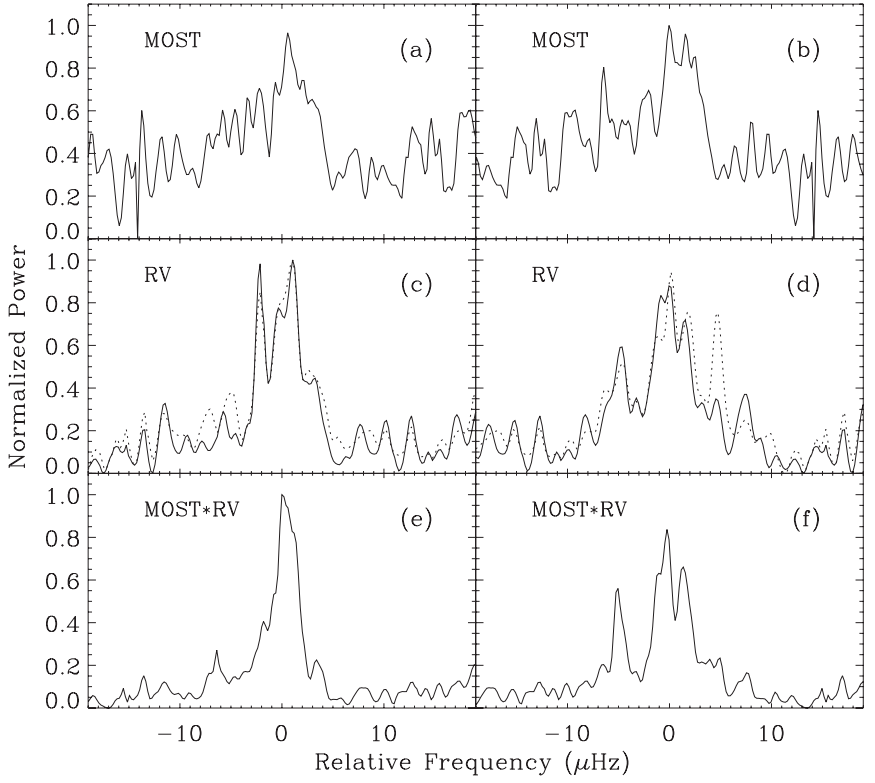

Figure 7. Collapsed échelle diagrams in the region $700-1300 \mu \mathrm{Hz}$ after correcting for curvature using ridge centroids. The top panels show the diagrams calculated using the MOST power spectrum, the middle panels using the radial velocity power spectrum, and the bottom panels using the product of both power spectra. Panels are separated into the left-hand side ridge (panels (a), (c), and (e)) and the right-hand side ridge (panels (b), (d), and (e)) as shown in Figure 6. The dotted lines in the middle panels show the radial velocity power spectrum collapsed over the full oscillation range (corresponding to Figure 10 in Bedding et al. 2010).

observations in intensity are less sensitive to pulsation modes of higher spherical degree than velocity measurements. We therefore expect ridges observed in photometry to be shifted to higher frequencies in the échelle diagram than in velocity, with the amount of shifting depending on the ridge identification. Calculations based on the theoretical response functions by Kjeldsen et al. (2008) and the frequency values presented in Bedding et al. (2010), however, showed that the expected shift is only of the order of $1 \mu \mathrm{Hz}$, too small to be detected with the current uncertainties.

Another possibility to search for ridge asymmetries is to increase the $\mathrm{S} / \mathrm{N}$ by collapsing the échelle diagram over several orders. To do so, we calculated the ridge centroids for the MOST data in the same manner as done by Bedding et al. (2010) for the radial velocities. Using the ridge centroids calculated for the MOST data, we straightened each order by removing the curvature seen in the échelle diagram before collapsing the échelle diagram in the frequency range where the centroids were reliably determined $(700-1300 \mu \mathrm{Hz})$. Figure 7 shows a comparison with the analogous procedure for the velocity power spectrum (see Figure 10 in Bedding et al. 2010) as well as for the product of the MOST and RV power spectrum. Note that the ridge centroids have been calculated for each of the three power spectra individually since, as explained above, it is expected that the ridge positions are slightly different for each data set. While the two ridges are clearly detected in the MOST photometry (top panels), the $\mathrm{S} / \mathrm{N}$ is too low to make any firm conclusion about the possible presence of separated ridges (i.e., $l=0$ and $l=2$ ), as is the case for the RV data (middle panels). We note, however, that the collapsed power spectrum of the combined data (bottom panels) might show some evidence for separated $l=0$ and $l=2$ components in the right-hand side ridge. This would imply Scenario A which is opposite to that preferred by Bedding et al. (2010), but in agreement with the results of Bayesian model comparisons using the RV data (Bedding et al. 2010; Handberg \& Campante 2011). A more detailed analysis including the extraction of individual frequencies from the combined data and a comparison with pulsation models will be presented in a forthcoming paper (T. Kallinger et al. 2011, in preparation).

\subsection{Oscillation Amplitudes}

\subsubsection{Amplitude Ratios}

The simultaneous observing campaigns allow us to measure the ratio of oscillation amplitudes in photometry and velocity and therefore test theoretical values and the scaling relations introduced by Kjeldsen \& Bedding (1995). The following influences have to be considered when measuring amplitudes of solar-like oscillations.

1. Systematic variations due to the stellar cycle effects.

2. Variations due to stochastic excitation and damping of the oscillation signal.

3. Measurement errors due to background subtraction.

Since both data sets have been obtained within a time span of less than 60 days, effects arising from (1) can be safely ignored here. To measure amplitudes in a way that is largely insensitive to point (2), we convolved the power spectrum with a Gaussian with FWHM $=4 \Delta v$ and scaled the signal to the contribution of radial modes in each order (Kjeldsen et al. 2008). Note that for the factor $c$, which measures the effective number of modes per order, we have interpolated the values listed in Table 1 of Kjeldsen et al. (2008) to the central wavelength of the MOST filter $(525 \mathrm{~nm})$, yielding $c=3.14$.

A crucial precondition to estimate the amplitude, particularly at low $\mathrm{S} / \mathrm{N}$, is to properly correct for the background contribution arising from stellar granulation and activity. In order to reliably estimate the uncertainty of the background parameters, we have used a combination of two published methods. An initial least-squares fit using the method of Huber et al. (2009a) was used as a starting point for a more careful fitting procedure using a Bayesian Markov-Chain Monte Carlo (MCMC) algorithm, as described in Gruberbauer et al. (2009) and Kallinger et al. (2010). The fitted model was adopted from Karoff (2008) and has the form

$$
P(\nu)=P_{n}+\sum_{i=0}^{k} \frac{4 \sigma_{i}^{2} \tau_{i}}{1+\left(2 \pi \nu \tau_{i}\right)^{2}+\left(2 \pi \nu \tau_{i}\right)^{4}},
$$

where $P_{n}$ is the white noise component, $k$ is the number of power laws used, and $\sigma$ and $\tau$ are the rms intensity and timescale of granulation, respectively. Note that in our application for Procyon, $k=2$.

The determination of the resulting background and corresponding amplitude was done in two steps.

1. We excluded the region of the power spectrum which contains oscillation signal and ran the Bayesian MCMC algorithm only on the remaining power spectrum. The excluded region was determined by visual inspection of heavily smoothed power spectra (see Figure 8), and we verified through several trial MCMC runs that changing this region within reasonable limits does not significantly influence the results. We used uniform priors for the granulation timescales and Jeffreys priors for the amplitudes 


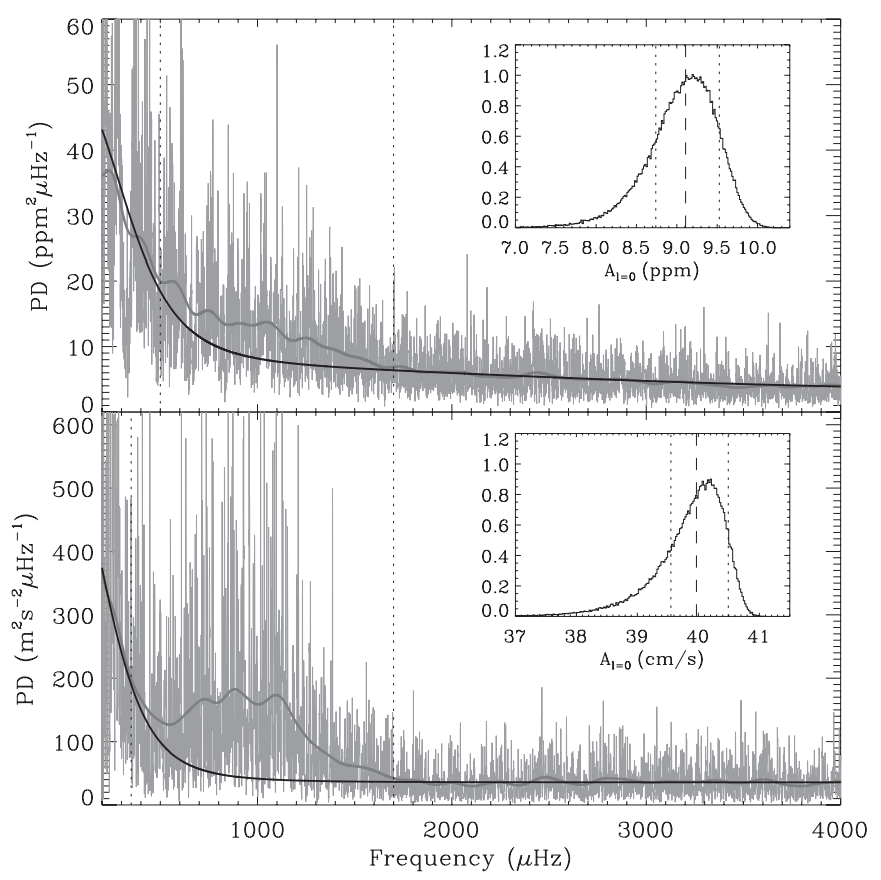

Figure 8. Power density spectra of Procyon smoothed with a $1 \mu \mathrm{Hz}$ boxcar (light gray) and $110 \mu \mathrm{Hz}$ boxcar (dark gray) observed in photometry (upper panel) and velocity (lower panel) with fitted background models (solid black lines). Vertical dotted lines mark the frequency intervals occupied by stellar oscillations which were excluded from the fit. The insets show the distributions of the mean amplitude per radial mode in the frequency interval 650-1150 $\mu \mathrm{Hz}$ derived from the MCMC simulations of the background fit. Dashed lines mark the median and dotted lines the $1 \sigma$ confidence limits.

of the background components. The most probable background model was then determined as the median of the marginalized posterior distributions for each background parameter.

2. Since the amplitude is not implicitly defined as a model parameter that is fitted to the data, we determined the pulsation amplitude as the mean level of the smoothed power spectrum in frequency range $650-1150 \mu \mathrm{Hz}$ after subtracting the background. This step was done for each MCMC iteration and the final amplitude was then evaluated as the median and $1 \sigma$ confidence limits of the resulting amplitude distribution.

Figure 8 shows the background fits resulting from 10 independent MCMC chains, each with $10^{5}$ iterations, for the full MOST and RV data sets. The distributions for the mean amplitude over the range of $650-1150 \mu \mathrm{Hz}$ (after subtracting the background signal) yields $A_{l=0 \text {,phot }}=9.1_{-0.4}^{-0.4} \mathrm{ppm}$ for the $M O S T$ data and $A_{l=0, \mathrm{RV}}=40.0_{-0.4}^{+0.5} \mathrm{~cm}^{-1} \mathrm{~s}$ for the velocity data. Hence, we arrive at a mean amplitude ratio for the full data sets of $A_{l=0, \text { phot }} / A_{l=0, \mathrm{RV}}=0.23 \pm 0.01 \mathrm{ppm} \mathrm{cm}^{-1} \mathrm{~s}$.

As shown by Kjeldsen et al. (2008) for the Sun, short mode lifetimes can cause considerable variations even after heavily smoothing the oscillation envelope. To test these effects, we employed a similar approach as Arentoft et al. (2008) and subdivided the MOST data into five independent subsets of equal length ( $\sim 7.5$ days). Note that this subset length is considerably longer than the 10 individual 2 day subsets used by Arentoft et al. (2008) for the radial velocity data since the signal in photometry is lower, requiring longer sets to achieve a sufficiently high signal for an amplitude determination. The resulting smoothed curves, which were corrected for a background fit calculated using $5 \times 10^{5} \mathrm{MCMC}$ iterations for each subset, together with their mean value are shown in Figure 9(a). Using the central region of maximum power between 650 and $1150 \mu \mathrm{Hz}$, we derived a mean amplitude of $A_{l=0 \text {,phot }}=9.1 \pm 0.5 \mathrm{ppm}$ in very good agreement with the value derived using the full data set above. In order to compare this photometric value to a velocity amplitude derived using the same method, we combine it with the estimate for the radial velocity amplitude given by Arentoft et al. (2008) as $A_{l=0, \mathrm{RV}}=38.1 \pm 1.3 \mathrm{~cm}^{-1} \mathrm{~s}$. This yields an amplitude ratio, measured using subsets, of $A_{l=0, \text { phot }} / A_{l=0, \mathrm{RV}}=0.24 \pm 0.02 \mathrm{ppm} \mathrm{cm}^{-1} \mathrm{~s}$, again in good agreement with the value derived from the full data sets.

A few points need to be considered when evaluating the quoted values and uncertainties. First, the fact that the uncertainty on $A_{l=0, \mathrm{RV}}$ derived from the full data set is considerably lower than the value derived by Arentoft et al. (2008) shows that for high $\mathrm{S} / \mathrm{N}$ data the influence of stochastic excitation (see point (2) above) dominates the amplitude uncertainty over the uncertainty arising from the background determination (see point (3) above). For the MOST data on the other hand, both uncertainties are roughly the same. To ensure a conservative approach, we therefore opted to use the amplitude ratio determined using subsets, $A_{l=0, \text { phot }} / A_{l=0, \mathrm{RV}}=0.24 \pm 0.02 \mathrm{ppm} \mathrm{cm}^{-1} \mathrm{~s}$, as our final value of the mean amplitude ratio.

Second, the amplitude ratios do not include any uncertainties arising from the factors $c$ used to normalize the amplitude per radial mode. First observational constraints on photometric mode visibility ratios by Deheuvels et al. (2010) suggest $\sim 2 \sigma$ differences of up to $25 \%$ compared to theoretical responses used by Kjeldsen et al. (2008). These differences could be caused by uncertainties in the limb-darkening laws used to calculate theoretical response functions, but also intrinsic differences in amplitudes of different degrees. We will assume that the latter cancel out in the photometry to velocity ratio, and therefore only concentrate on the mode visibilities. We repeated the calculations of $c$ by numerically integrating the spatial response functions for a quadratic limb-darkening law for the Sun (Bedding et al. 1996) assuming a conservative absolute uncertainty of 0.05 (corresponding to a relative uncertainty of $\sim 10 \%-20 \%$ ) for each limb-darkening coefficient (Howarth 2010). The resulting $c$ factors after 1000 integrations yield an uncertainty of $4 \%$ in velocity and $6 \%$ in photometry, which translates into uncertainties of $2 \%$ and $3 \%$ in the normalized amplitudes and hence an uncertainty of $4 \%$ in the amplitude ratio. This test shows that the uncertainty on $c$ can be substantial for estimating amplitude ratios, in particular for observations with high $\mathrm{S} / \mathrm{N}$ and long mode lifetimes as found in red giant stars (De Ridder et al. 2009). In our case, however, the amplitude ratio is dominated by the $\sim 8 \%$ uncertainty arising from the background fits and finite mode lifetimes.

\subsubsection{Comparison with Theoretical Results}

How do our estimates for the amplitude ratio compare with scaling relations? Rearranging Equation (5) in Kjeldsen \& Bedding (1995) yields

$$
\frac{(\delta L / L)_{\lambda} / \mathrm{ppm}}{v_{\mathrm{osc}} / \mathrm{cm} \mathrm{s}^{-1}}=\frac{20.1 \times 10^{-3}}{(\lambda / 550 \mathrm{~nm})\left(T_{\mathrm{eff}} / 5777 \mathrm{~K}\right)^{r}},
$$

with $r=1.5$ if the oscillations are adiabatic and a best-fitting value of $r=2$ for observed amplitudes in classical pulsators (see Kjeldsen \& Bedding 1995).

Using $r=2, T_{\text {eff }}=6500 \mathrm{~K}$, and a wavelength $\lambda=$ $525 \mathrm{~nm}$, the expected amplitude ratio for radial modes is 


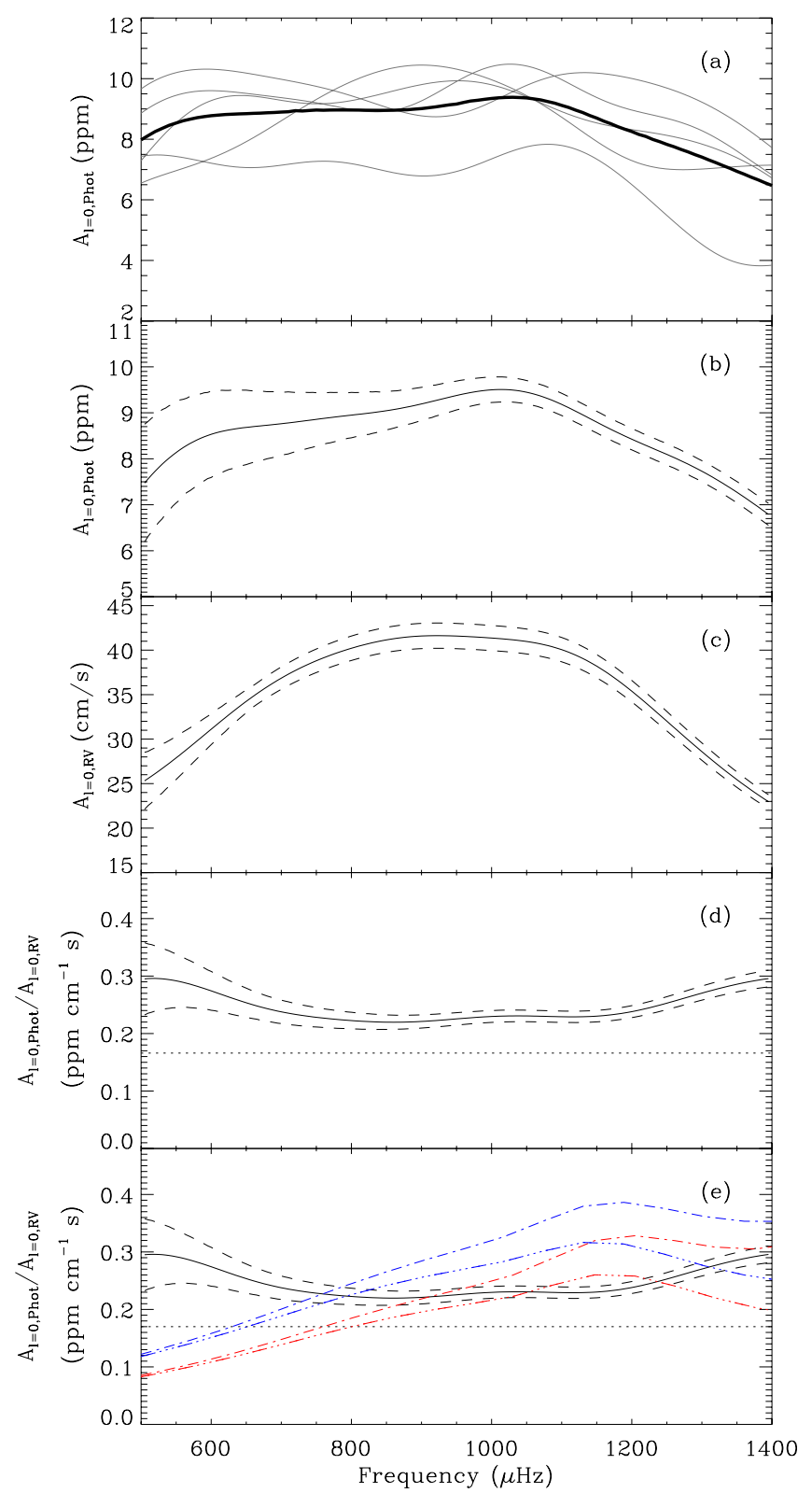

Figure 9. (a) Smoothed amplitude curves of five independent 7.5 day subsets of MOST photometry (thin lines) together with their mean. (b) Smoothed amplitude curve using the full MOST data set. Dashed lines mark the $1 \sigma$ uncertainties. (c) Same as panel (b) but for the full RV time series. (d) Ratio of the curves shown in panels (b) and (c). The horizontal dotted line shows the expected amplitude ratio using the scaling relation of Kjeldsen \& Bedding (1995). (e) Same as panel (d), but compared to theoretical amplitude ratios using an Eddington atmosphere (red lines) and a scaled VAL-C atmosphere (blue lines), for heights of $300 \mathrm{~km}$ (dashed-dotted lines) and $600 \mathrm{~km}$ (dashed-triple-dotted lines) above the photosphere (see Houdek 2010, for details).

(A color version of this figure is available in the online journal.)

$0.17 \mathrm{ppm} \mathrm{cm}^{-1}$ s. As shown by the dotted line in Figure 9(d), this is considerably lower than the observed ratio. Using $r=1.5$ the agreement is only slightly better, with an expected ratio of $0.18 \mathrm{ppm} \mathrm{cm}^{-1} \mathrm{~s}$. Since the RV amplitude is in agreement with the value from scaling relations (see Arentoft et al. 2008), this implies that the MOST amplitude is higher than expected.

Going one step further, the smoothed amplitude curves allow us to analyze the amplitude ratio as a function of frequency. Figure 9(d) shows the ratio of the amplitude curves derived from the full data sets for MOST and the RV data which are shown separately in Figure 9(b) and (c). Note that we have scaled the uncertainty of the RV amplitude curve to match the relative uncertainty derived using subsets, as described in the previous section. We also repeated the calculation using only HARPS data from the RV data set and restricted the MOST data to the same time span $(\sim 6.5$ days $)$. The result was almost identical to the result based on the full data set but with larger uncertainty.

Figure 9(e) compares the amplitude ratio as a function of frequency with theoretical predictions by Houdek (2010) for a model of Procyon for different model atmospheres and different heights above the photosphere. Although the exact shape of the variation as a function of frequency is not well recovered, we note that the average amplitude ratio at maximum power $(\sim 1000 \mu \mathrm{Hz})$ is in better agreement with models than the estimate based on scaling relations (dotted line). A more detailed comparison will have to await the collection of higher $\mathrm{S} / \mathrm{N}$ data from which amplitudes of individual mode frequencies can be reliably extracted.

It is interesting to note that, as can be seen from Figures 6, 8,9 (b) and (c), the maximum of the oscillation envelope in photometry seems to be shifted to slightly higher frequencies than in velocity. Defining $v_{\max }$ as the frequency corresponding to the maximum of the smoothed oscillation envelope, the MCMC analysis of the full data sets yields $v_{\text {max,phot }}=1014_{-11}^{+8} \mu \mathrm{Hz}$, compared to $v_{\max , \mathrm{RV}}=923_{-11}^{+9} \mu \mathrm{Hz}$. This shift translates into an increase of the amplitude ratio as a function of frequency, which tentatively can be identified in Figure 9(d).

\section{CONCLUSIONS}

We have compared simultaneous space-based MOST photometry and ground-based RV data of the F5 star Procyon. Our main findings can be summarized as follows.

1. The MOST light curves of 2004, 2005, and 2007 show slow variations similar to those observed in velocity. A comparison of the variability level in photometry and velocity to the Sun confirmed that these variations are compatible with stellar activity on Procyon.

2. The peaks observed in the MOST 2007 power spectrum match the oscillation frequencies detected in the RV campaign, and the échelle diagrams show similar structure and curvature. We have attempted to confirm the mode ridge identification presented by Bedding et al. (2010) by collapsing power spectra corrected for curvature. While the MOST data alone do not provide conclusive results, the collapsed échelle diagram of the combined data sets shows some evidence that Scenario A in Bedding et al. (2010) is the correct mode identification, contradicting the conclusions of that paper. Further work based on the extraction of individual frequencies from the combined data and a comparison with stellar models will be necessary to confirm this result.

3. We measured the mean luminosity amplitude per radial mode in Procyon in the frequency range $650-1150 \mu \mathrm{Hz}$ to be $A_{l=0 \text {,phot }}=9.1 \pm 0.5 \mathrm{ppm}$, in agreement with the value of $8.5 \pm 2$ ppm published by Bruntt et al. (2005). Combining this with the mean velocity amplitude measured by Arentoft et al. (2008) gives an amplitude ratio of $A_{l=0, \text { phot }} / A_{l=0, \mathrm{RV}}=$ $0.24 \pm 0.02 \mathrm{ppm} \mathrm{cm}^{-1} \mathrm{~s}$. This is considerably higher than the value of $0.17 \mathrm{ppm} \mathrm{cm}^{-1} \mathrm{~s}$ expected from scaling from the Sun, but is in better agreement with theoretical values predicted by Houdek (2010). We also analyzed the 
amplitude ratio as a function of frequency and found that the maximum of the oscillation envelope appears to be shifted to higher frequencies in photometry than in velocity.

The results presented here illustrate the potential of combining simultaneous luminosity and velocity measurements to study pulsations in stars. Future opportunities may arise from combining measurements from the ground-based RV network SONG (Grundahl et al. 2008) with space-based photometry by MOST and BRITE (Weiss et al. 2008), which will mark an important step in studying stellar structure and evolution in bright stars with well-known fundamental parameters.

The authors dedicate this publication to the memory of our dear friend and colleague Dr. Piet Reegen, who sadly left us before his time. We are thankful to our anonymous referee for very helpful comments which improved this manuscript. D.H. acknowledges support by the Astronomical Society of Australia (ASA). T.R.B. and D.S. acknowledge support by the Australian Research Council. G.H. acknowledges support by the Austrian FWF Grant No. P2120521. T.K. and W.W.W. are supported by the Austrian Fonds zur Förderung der wissenschaftlichen Forschung (FWF), project number P22691-N16. The Austrian participation in the MOST project is funded by the Austrian Research Promotion Agency (FFG). M.G., D.B.G., and J.M.M. acknowledge funding from the Natural Sciences \& Engineering Research Council (NSERC) Canada.

\section{REFERENCES}

Allende Prieto, C., Asplund, M., García López, R. J., \& Lambert, D. L. 2002, ApJ, 567, 544

Arentoft, T., et al. 2008, ApJ, 687, 1180

Barban, C., et al. 1999, in ASP Conf. Ser. 185, IAU Colloq. 170, Precise Stellar Radial Velocities, ed. J. B. Hearnshaw \& C. D. Scarfe (San Francisco, CA: ASP), 177

Basri, G., et al. 2010, ApJ, 713, L155

Baudin, F., Appourchaux, T., Boumier, P., Kuschnig, R., Leibacher, J. W., \& Matthews, J. M. 2008, A\&A, 478, 461

Bedding, T. R., Kjeldsen, H., Reetz, J., \& Barbuy, B. 1996, MNRAS, 280, 1155

Bedding, T. R., et al. 2005, A\&A, 432, L43

Bedding, T. R., et al. 2010, ApJ, 713, 935

Benomar, O., et al. 2009, A\&A, 507, L13

Bouchy, F., Maeder, A., Mayor, M., Mégevand, D., Pepe, F., \& Sosnowska, D. 2004, Nature, 432, 2

Brown, T. M., Gilliland, R. L., Noyes, R. W., \& Ramsey, L. W. 1991, ApJ, 368, 599

Bruntt, H., Kjeldsen, H., Buzasi, D. L., \& Bedding, T. R. 2005, ApJ, 633, 440

Chaplin, W. J., Houdek, G., Karoff, C., Elsworth, Y., \& New, R. 2009, A\&A, 500, L21

Clarke, D. 2003, A\&A, 407, 1029

Claudi, R. U., et al. 2004, Commun. Asteriseismol., 145, 53

Croll, B., et al. 2006, ApJ, 648, 607
De Ridder, J., et al. 2009, Nature, 459, 398

Deheuvels, S., et al. 2010, A\&A, 515, A87

Dorren, J. D. 1987, ApJ, 320, 756

Eggenberger, P., Carrier, F., Bouchy, F., \& Blecha, A. 2004, A\&A, 422, 247

Fröhlich, C., et al. 1997, Sol. Phys., 170, 1

García, R. A., et al. 2005, A\&A, 442, 385

Gelly, B., Gree, G., \& Fossat, E. 1986, A\&A, 164, 383

Gilliland, R. L., et al. 2010, PASP, 122, 131

Grec, G., Fossat, E., \& Pomerantz, M. A. 1983, Sol. Phys., 82, 55

Gruberbauer, M., Kallinger, T., Weiss, W. W., \& Guenther, D. B. 2009, A\&A, 506,1043

Grundahl, F., Christensen-Dalsgaard, J., Kjeldsen, H., Frandsen, S., Arentoft, T., Kjaergaard, P., \& Jørgensen, U. G. 2008, in IAU Symp. 252, The Art of Modeling Stars in the 21 st Century, ed. L. Deng \& K. L. Chan (Cambridge: Cambridge Univ. Press), 465

Guenther, D. B., et al. 2007, Commun. Asteroseismol., 151, 5

Guenther, D. B., et al. 2008, ApJ, 687, 1448

Handberg, R., \& Campante, T. L. 2011, A\&A, 527, A56

Houdek, G. 2010, Ap\&SS, 328, 237

Howarth, I. D. 2010, arXiv:1011.2631

Huber, D., Stello, D., Bedding, T. R., Chaplin, W. J., Arentoft, T., Quirion, P., \& Kjeldsen, H. 2009a, Commun. Asteroseismol., 160, 74

Huber, D., et al. 2009b, A\&A, 505, 715

Innis, J. L., Isaak, G. R., Speake, C. C., Williams, H. K., \& Brazier, R. I. 1991, MNRAS, 249, 643

Kallinger, T., et al. 2010, A\&A, 509, A77

Karoff, C. 2008, PhD thesis, Univ. Aarhus

Kjeldsen, H., \& Bedding, T. R. 1995, A\&A, 293, 87

Kjeldsen, H., et al. 2008, ApJ, 682, 1370

Lanza, A. F., Rodonò, M., Pagano, I., Barge, P., \& Llebaria, A. 2003, A\&A, 403, 1135

Lanza, A. F., et al. 2009, A\&A, 493, 193

Lanza, A. F., et al. 2011, A\&A, 525, A14

Leccia, S., Kjeldsen, H., Bonanno, A., Claudi, R. U., Ventura, R., \& Paternò, L. 2007, A\&A, 464, 1059

Libbrecht, K. G. 1988, in IAU Symp. 132, The Impact of Very High S/N Spectroscopy on Stellar Physics, ed. G. Cayrel de Strobel \& M. Spite (Dordrecht: Kluwer), 83

Marchenko, S. V. 2008, A\&A, 479, 845

Martić, M., Lebrun, J., Appourchaux, T., \& Korzennik, S. G. 2004, A\&A, 418, 295

Martić, M., et al. 1999, A\&A, 351, 993

Matthews, J. M. 2007, Commun. Asteroseismol., 150, 333

Matthews, J. M., Kusching, R., Guenther, D. B., Walker, G. A. H., Moffat, A. F. J., Rucinski, S. M., Sasselov, D., \& Weiss, W. W. 2004, Nature, 430, 51

Michel, E., Samadi, R., Baudin, F., Barban, C., Appourchaux, T., \& Auvergne, M. 2009, A\&A, 495, 979

Michel, E., et al. 2008, Science, 322, 558

Mosser, B., Baudin, F., Lanza, A. F., Hulot, J. C., Catala, C., Baglin, A., \& Auvergne, M. 2009, A\&A, 506, 245

Mosser, B., Maillard, J. P., Mekarnia, D., \& Gay, J. 1998, A\&A, 340, 457

Reegen, P., et al. 2006, MNRAS, 367, 1417

Régulo, C., \& Roca Cortés, T. 2005, A\&A, 444, L5

Ulrich, R. K., et al. 2000, A\&A, 364, 799

Walker, G., et al. 2003, PASP, 115, 1023

Walker, G. A. H., et al. 2007, ApJ, 659, 1611

Weiss, W. W., Moffat, A. F. J., \& Kudelka, O. 2008, Commun. Asteroseismol., 157,271 